\title{
Herpes Zoster Associated Hospital Admissions in Italy: Review of the Hospital Discharge Forms
}

\section{Giovanni Gabutti ${ }^{1}$, , Carlotta Serenelli ${ }^{1}$, Alessandra Cavallaro ${ }^{1}$ and Pietro Ragni ${ }^{2}$}

1 Department of Clinical and Experimental Medicine, Section of Hygiene and Occupational Health, University of Ferrara; Via Fossato di Mortara 64b, 44100 Ferrara, Italy

2 Health Management, LHU of Reggio Emilia; Via Amendola 2, 42100, Reggio Emilia, Italy

* Author to whom correspondence should be addressed; E-Mail: giovanni.gabutti@unife.it; Tel.: +39-532-45-55-68; Fax: +39-532-20-50-66.

Received: 22 July 2009 / Accepted: 26 August 2009 / Published: 2 September 2009

\begin{abstract}
In Italy a specific surveillance system for zoster does not exist, and thus updated and complete epidemiological data are lacking. The objective of this study was to retrospectively review the national hospital discharge forms database for the period 19992005 using the code ICD9-CM053. In the period 1999-2005, 35,328 hospital admissions have been registered with annual means of 4,503 hospitalizations and 543 day-hospital admissions. The great part of hospitalizations (61.9\%) involved subjects older than 65 years; the mean duration of stay was 8 days. These data, even if restricted to hospitalizations registered at national level, confirm the epidemiological impact of shingles and of its complications.
\end{abstract}

Keywords: herpes zoster; hospital admissions; epidemiology

\section{Introduction}

Varicella (chickenpox) is a highly contagious infectious disease caused by an alpha-herpes virus: the Varicella Zoster Virus (VZV). From the epidemiological point of view, man is the only reservoir of infection and VZV spreads from person to person via droplet nuclei or via direct contact with skin 
lesions. The virus has a global distribution and a typical endemic-epidemic course. The infection mostly affects childhood and the higher incidence rates are registered in the 0-14 years age class [1].

In Italy universal mass vaccination against varicella has not been adopted and, for this reason, VZV continues to spread, causing each year a number of cases corresponding to a birth cohort [2].

During primary infection, the etiological agent has the ability to infect the sensory nerves in mucocutaneous sites and then to become latent in the sensory-nerve ganglia [3]. In detail, after replication at the portal of entry there is a viremia and the spreading of the virus to skin and mucosae. Afterwards, there is a new replicative phase causing both the typical cutaneous rash and the infection of sensory nerves in the epithelium. From the sensory nerves the virus reaches the sensory ganglia were it becomes latent. The reactivation of the latent virus, years or decades after primary infection, causes the typical clinical expression called Zoster.

From the immunological point of view, VZV elicits a long-lasting response against the clinical expression (varicella or chickenpox); however, naturally acquired immunity neither prevents virus latentization nor its possible reactivation. Natural infection stimulates both humoral and cell-mediated immunity (CMI) [4]. Nowadays, CMI is considered extremely important, as VZV reactivation is believed as strictly related to a decrease of specific T-lymphocytes [5]. An episode of zoster elicits a restore of CMI and usually recurrent episodes of shingles are rare.

During life-time the risk of an episode of zoster is estimated equal to $10-30 \%$ and it is strictly age-related. Incidence increases sharply with aging; about $50 \%$ of subjects aged 80 years or older are affected by at least one episode of zoster [6].

From the clinical point of view, in the immunocompetent patient zoster is characterized by a dermatomal vesicular rash, lasting about 2-3 weeks and accompanied by moderate to severe pain. The clinical picture can involve any dermatome, but the preferred sites are the thoracic or lumbar nerve segments and the distribution area of the trigeminal nerve. Usually a prodromic phase, lasting a few days (1-5), foregoes the clinical expression of the disease; this is characterized by pain, even burning, hitch, paraesthesia, etc. In the immunocompetent subject the vesicular phase lasts about one week; afterwards, blisters dry out and turn into crusts which fall off after 2-4 weeks [7]. In some patients mild forms, even without rash (zoster sine herpete), can occur. An important number of patients experiences a long-lasting pain that does not end with the disappearance of the rash but persists for some weeks, and even months or years. This chronic neuropathic pain is defined as post-herpetic neuralgia (PHN). PHN is an important cause of stress and disability and contributes significantly to the worsening of patients' quality of life [8,9]. PHN is estimated to occur in $10-20 \%$ of zoster cases; differences in the incidence rate are related to the different definitions of PHN and to the age of patients [10]. As a matter of fact, the incidence of PHN increases with age. In immunocompromised patients, particularly in those affected by cellular immunodeficiency, zoster can be clinically more severe, with atypical presentations, forms with necrosis and involvement of internal organs [11]. The decay of CMI and aging are considered risk factors for the development of zoster. However, even in immunocompetent subjects, intrauterine exposure and VZV acquisition before 18 months of age are related to an increased risk. Other risk factors, such as gender, seasonality, exposure to immunotoxic chemical substances, mechanical traumas and genetic susceptibility have not been clearly related to zoster development [6]. 
In Italy, a specific surveillance system for zoster does not exist and therefore complete and updated epidemiological data are not available at a national level. Notification of zoster cases is usually not performed and existing data have been obtained by research performed in different areas and with different methodologies. At a national level, there is the possibility to evaluate the hospital discharge forms (SDO) which represent an official instrument of the Italian Ministry of Health containing clinical and organizational information related to the hospitalizations [12]. The purpose of this study was to provide updated epidemiological data, describing the trend of the hospitalizations for zoster in Italy through a search in the national SDO database.

\section{Methods}

\subsection{Background}

The SDO database was officially established in 1991 [13]; each form is a concise and accurate summary of the case history. It allows one to collect in a systematic and economic way the main information included in the case history and to check their quality later. Each SDO provides information about every patient discharged from all public and private hospitals nationwide and is filled in by the doctors who treated the patient. Information regard both clinical (diagnosis, symptoms, surgical interventions, diagnostic-therapeutic procedures, prosthetic implantations, methods of discharge) and organizational aspects (operative unit of admission and discharge, transfers, who pays for admission costs, etc.). The clinical information is coded by the international ICD9-CM system (International Classification of Diseases, $9^{\text {th }}$ revision, Clinical Modification), currently used in Italy [14]. The SDO are then coded and transmitted to the Regions and to the National Ministry of Health to be included in the regional and national SDO database.

\subsection{Study Design}

In this research, the national database was retrospectively searched over the period 1999-2005 using the code ICD9-CM 053. Noteworthy, in the regional SDO only the main diagnosis is included and cases are ordered by solar year and age groups. The search can be performed in the age classes: 0-14 years, 15-64 years and >64 years; a further subdivision of these groups is not available. Data related to each single hospitalization/day hospital admission are not available; for this reason some statistical analysis (e.g., comparison between average length of stay, etc.) were not possible.

In detail, we searched for hospitalizations due to zoster (ICD code 053), herpes zoster with meningitis (ICD 0530), herpes zoster with unspecified complications of the nervous system (ICD 05310), Ramsay Hunt syndrome (ICD 05311), trigeminal post-herpetic neuralgia (ICD 05312), postherpetic polyneuropathy (ICD 05313), herpes zoster with other complications of the nervous system (ICD 05319), eyelid dermatitis due to herpes zoster (ICD 05320), keratoconjunctivitis due to herpes zoster (ICD 05321), uveitis due to herpes zoster (ICD 05322), herpes zoster with other ophtalmic complications (ICD 05329), otitis externa due to herpes zoster (ICD 05371), herpes zoster with other specified complications (ICD 05379), herpes zoster with unspecified complications (ICD 0538), herpes 
zoster without complications (ICD 0539). Statistical analysis was performed by StatView software version 5.0; differences among percentages were assessed by the chi-square test.

\section{Results}

In the period 1999-2005, 35,328 hospitalizations for zoster have been registered in total: 31,526 hospitalizations and 3,802 day-hospital admissions. The annual mean was equal to 4,503 hospitalizations and 543 day-hospital admissions; these data correspond to 14 admissions per day. The annual incidence of HZ hospitalizations (day hospital admissions included) per 1,000 inhabitants ranged between 0.070 and $0.1 / 1,000$ (respectively in 2005 and 1999).

In the examined period a gradual and significant decrease of hospitalizations was observed $(5,383$ in 1999, 3,536 in 2005); however, a significant increase of day-hospital admissions was registered. These were 507 in 1999 and 558 in 2005, representing the $8.6 \%$ and the $13.6 \%$ of hospitalizations due to HZ in the same years, respectively (chi square $=117.036 ; \mathrm{p}<0.01$ ). In the same period, the analysis of admissions for any cause showed an analogous, even if more evident, trend; there was a gradual and significant decrease of hospitalizations (from 10,165,184 in 1999 to 8,970,561 in 2005) and a corresponding increase of day-hospital admissions (2,502,993 in 1999 and 3,984,338 in 2005) (chi square $=70822.219 ; \mathrm{p}<0.01)$.

The analysis of SDO data, stratified according to gender, has always shown a greater number of cases in females; the 55\% of hospitalizations due to herpes zoster in the period 1999-2005 involved this gender. Moreover, the predominance of female gender is clear, analyzing the stratification of both Italian population (chi square $=322.621 ; \mathrm{p}<0.0001$ ) and hospitalizations due to any cause (chi square $=602.602 ; \mathrm{p}<0.001)$ (Table 1).

Hospitalizations due herpes zoster stratified according to age classes showed an increase with aging; in the period $1999-2005$, the $61.9 \%$ of hospitalizations has involved subjects older than 65 years $(26 \%$ in $65-74$ yrs age group and $35.9 \%$ in $\geq 75$ yrs. age group) (Figure 1).

The average length of hospital stay, evaluated only for hospitalizations, was 8 days. This value resulted greater in older patients: 6.8 days and 8.8 days in subjects $<65$ yrs. and $>65$ yrs, respectively.

Table 1. Resident population and hospitalizations due to all causes and herpes zoster, Italy 1999-2005.

\begin{tabular}{|c|c|c|c|}
\hline Year & Males & Females & Total \\
\hline \multicolumn{4}{|c|}{ Resident population } \\
\hline 1999 & $27,563,748$ & $29,345,361$ & $56,909,109$ \\
\hline 2000 & $27,562,988$ & $29,360,536$ & $56,923,524$ \\
\hline 2001 & $27,576,326$ & $29,384,366$ & $56,960,692$ \\
\hline 2002 & $27,587,242$ & $29,406,500$ & $56,993,742$ \\
\hline 2003 & $27,766,223$ & $29,554,847$ & $57,321,070$ \\
\hline 2004 & $28,068,608$ & $29,819,637$ & $57,888,245$ \\
\hline 2005 & $28,376,804$ & $30,085,571$ & $58,462,375$ \\
\hline
\end{tabular}


Table 1. Cont.

\begin{tabular}{|l|l|l|l|}
\hline \multicolumn{3}{|l|}{ Hospitalizations due to all causes } \\
\hline 1999 & $5,963.752$ & $6,704,425$ & $12,668,177$ \\
2000 & $5,870,813$ & $6,679,844$ & $12,550,657$ \\
2001 & $6,047,742$ & $6,873,203$ & $12,920,945$ \\
2002 & $6,039,504$ & $6,891,981$ & $12,931,485$ \\
2003 & $5,989,023$ & $6,814,980$ & $12,804,003$ \\
2004 & $6,054,139$ & $6,925,270$ & $12,979,409$ \\
2005 & $6,051,244$ & $6,903,655$ & $12,954,899$ \\
\hline Hospitalizations due to herpes zoster & \\
\hline 1999 & 2,635 & 3,255 & 5,890 \\
2000 & 2,522 & 3,099 & 5,621 \\
2001 & 2,472 & 3,012 & 5,484 \\
2002 & 2,330 & 2,879 & 5,209 \\
2003 & 2,163 & 2,524 & 4,687 \\
2004 & 1,904 & 2,439 & 4,343 \\
2005 & 1,873 & 2,221 & 4,094 \\
\hline
\end{tabular}

Figure 1. Herpes zoster: hospitalizations and day-hospital admissions stratified by age classes, Italy 1999-2005.

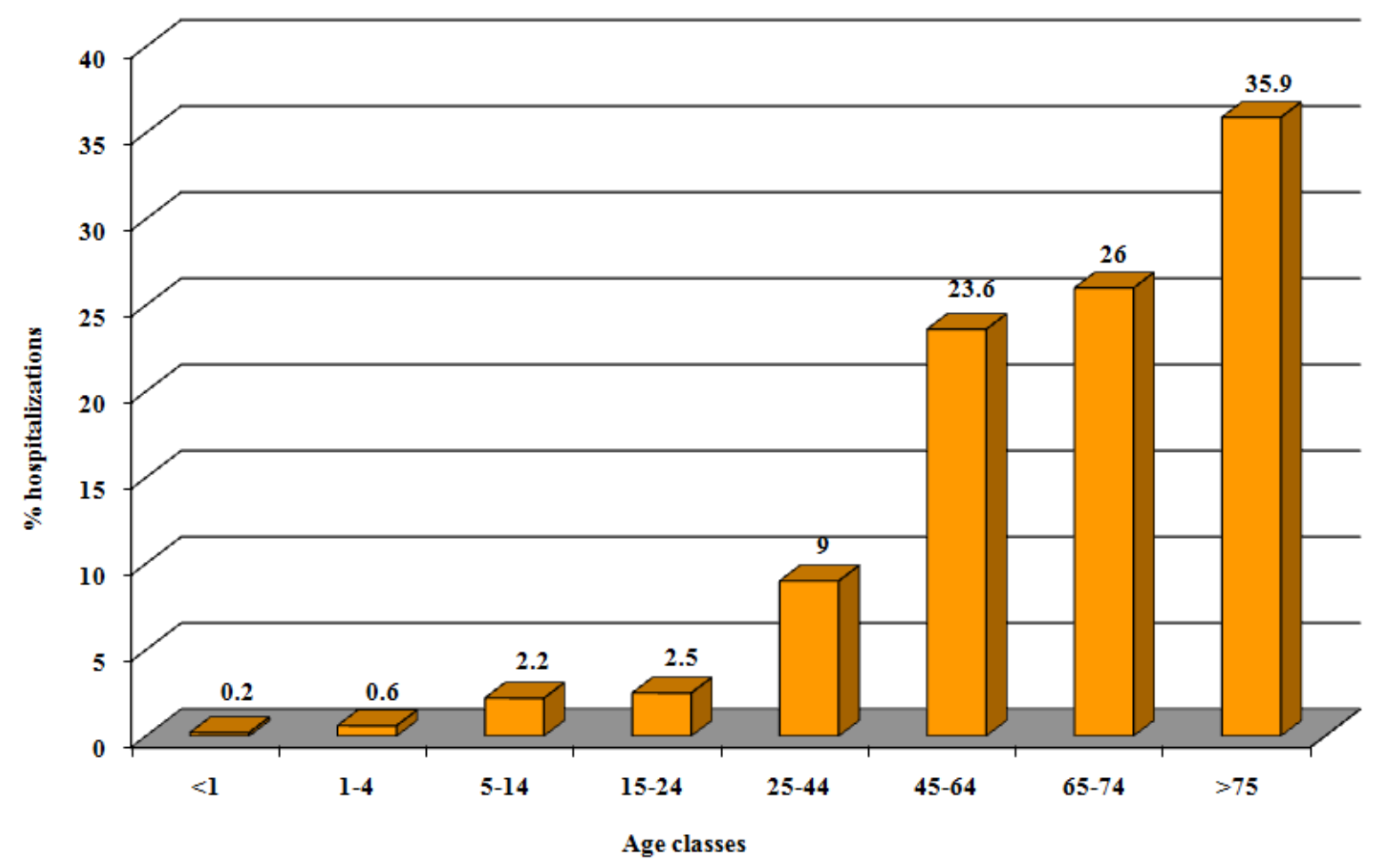


Besides, in order to perform a thorough investigation, the different ICD9-CM codes of the different subgroups included in the code 053 , corresponding to zoster, were considered. The code ICD 0539 (herpes zoster without complications) included $52 \%$ of all hospital admissions due to zoster in the observed period; the $60 \%$ of these cases involved patients older than 65 years. The remaining $48 \%$ of cases was equally distributed between the remaining codes, which include zoster complications (Figure 2).

Figure 2. Herpes zoster: hospitalizations stratified by ICD9CM code, Italy 1999-2005.

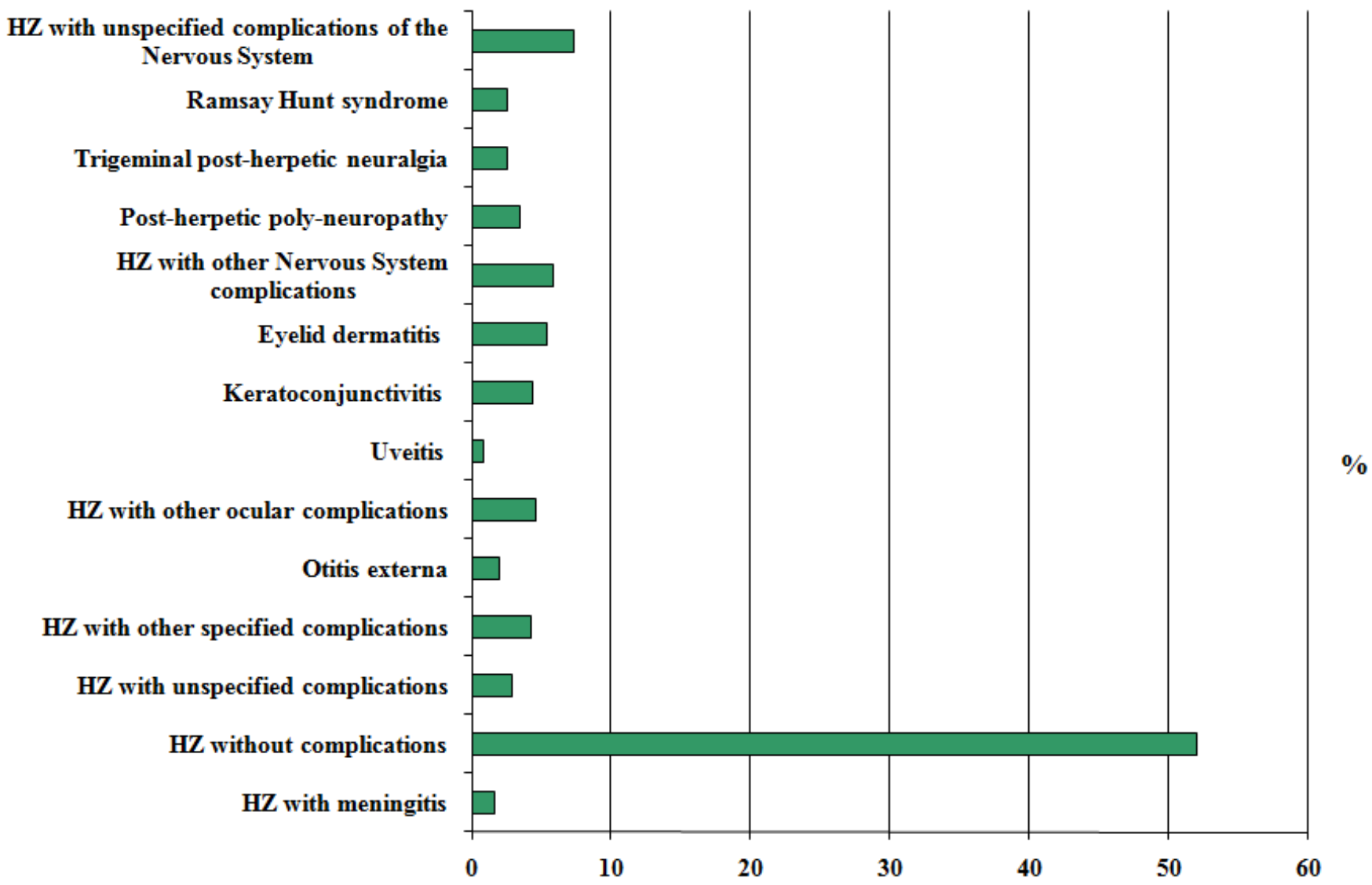

In detail, ocular complications identified by the codes ICD 05321 (keratoconjunctivitis due to zoster), 05322 (uveitis due to zoster), 05329 (herpes zoster with other ocular complications), represented the $9.8 \%$ of total hospital admissions due to zoster (mean length of stay: 8 days). Neurological complications, including the codes ICD9-CM 0530 (herpes zoster with meningitis), 05310 (herpes zoster with unspecified complications of the nervous system), 05311 (Ramsay Hunt syndrome), 05312 (trigeminal post-herpetic neuralgia), 05313 (post-herpetic polyneuropathy), 05319 (herpes zoster with other nervous system complications), represented the $23.5 \%$ of all hospitalizations due to zoster in the period 1999-2005. The mean length of stay of these cases was 10 days. The remaining 15\% of hospital admissions included the following ICD codes: 0538 (herpes zoster with unspecified complications), 05320 (eyelid dermatitis due to zoster), 05371 (otitis externa due to zoster), 05379 (herpes zoster with other specified complications). 


\section{Discussion}

The mandatory notification system adopted at national level for infectious diseases includes zoster among diseases notifiable in the 5th Class [15]. Many diseases included in this group are erroneously considered not subject to notification and, for this reason, adequate and updated epidemiological data are missing. This also happens for zoster, and the estimates of the impact of this disease come from studies conducted in different areas and with different methodologies. For example, a retrospective research performed at national level in 1996 involving dermatologists, geriatric doctors and general practitioners provided an estimate that each year in Italy about 200,000 cases of zoster and 42,000 cases of PHN occur in subjects older than $15 \mathrm{yrs}$. According to this study, the estimated incidence of zoster was equal to 4.14 cases per 1,000 subjects $\geq 15$ yrs.; the $45.8 \%$ of cases were registered in subjects older than $65 \mathrm{yrs}$. (47.1\% in subjects $>55 \mathrm{yrs}$.) and the $44.2 \%$ of cases involved still working subjects. The mean duration of each case was 11-15 days and complications occurred in the $26.1 \%$ of cases. PHN came out as the most important and frequent complication (26.1\%); antiviral therapy was prescribed for almost every patient [16]. Another study, performed in the Latium Region, took into account direct and indirect costs and estimated the overall costs of a zoster case equal to $987.00 €$ [17]. More recently, an observational prospective research performed in the Piedmont Region evaluated all cases of zoster diagnosed by a group of general practitioners. The annual incidence was 1.74 cases per 1,000 subjects older than 14yrs.; the rate standardized by age in the same group was 1.59 per 1,000 subjects. The rate of hospital admission was $0.12 / 1,000$ inhabitants. The mean cost of each domiciliary case and hospital admission was equal to $136.06 €$ and $4082.59 €$, respectively [18].

The data obtained in the present study, even if exclusively related to national hospitalizations, confirm the epidemiological impact of zoster and of its complications. This pathology involves all age groups; however, as observed in other studies, the disease is more frequent in older subjects [19]. With aging, there is an increase of both incidence and mean duration of hospital stay; this one is strictly related to the increased co-morbidity typical of older people. The higher incidence rates in the female population are related to the fact that old women are numerically prevalent on the old men, as can be drawn analyzing the stratification by gender of both the Italian population and the overall hospital admissions.

Considering the different ICD9-CM codes, 50\% of cases are identified as herpes zoster without complications. The hospital admissions identified as cases with complications were of neurological $(23.5 \%)$ and ocular (9.8\%) pertinence. Noteworthy, cases with neurological complications showed a longer length of stay (10 days vs 8 days of other admissions).

The impact of a disease should be evaluated also from an economic perspective. One method for the determination of costs related to hospitalizations is the evaluation of Diagnosis Related Groups (DRG) rates. According to the DRG reimbursement system, every hospitalized patient belongs to a group of cases diagnostically homogeneous; therefore, patients within each category are clinically similar and are expected to use the same level of hospital resources. For these reasons, patients in the same DRG group are assigned to the same reimbursement charges. In case of outliers (patients with a length of stay value higher or lower to that individually established for every DRG), the cost-weight increases or decreases for every day more or less compared to the average length of stay. A specific DRG for zoster does not exist. According to the great variability of the different DRGs that could be assigned to 
hospital admissions for zoster (range 887.32-4740.17€), it is quite difficult to estimate the economic impact of hospitalized cases.

All these data confirm the great impact of herpes zoster in the population [20]. Besides, treatment options for zoster and its complications are often sub-optimal and this fact implies a bad quality of patient's life [21,22]. The new knowledge on zoster etiopathogenesis and the compelling need to prevent this disease have prompted researchers to evaluate the possibility to reduce the incidence and severity of zoster and of its complications boosting CMI. In the last decade it has been demonstrated that varicella vaccines with a high antigenic content could elicit a significant increase of CMI in old immunocompetent subjects [23]. A "zoster vaccine", with a mean potency of 24,600 PFU (an antigenic content 14 times greater than the paediatric formulation of varicella vaccine), has been developed. A clinical trial performed in subjects $>60$ yrs of age allowed to demonstrate that this vaccine is safe, well tolerated and able to impact on the disease decreasing the burden of the illness $(61.1 \%)$, the incidence of zoster (51.3\%) and the incidence of PHN (66.5\%) [24]. The data of this clinical trial showed that a "zoster vaccine" can significantly boost CMI and is an interesting opportunity of preventive intervention against zoster and its complications [25]. Recently, the Advisory Committee on immunization practices (ACIP) has provided recommendations for the use of the already commercially available "zoster vaccine" among adults aged $\geq 60$ years in the United States [26].

\section{Acknowledgements}

The authors declare that they have no conflicts of interest.

\section{References}

1. Hambleton, S.; Gershon, A.A. Preventing Varicella-Zoster disease. Clin. Microbiol. Rev. 2005, 18, 70-80.

2. Gabutti, G.; Rota, M.C.; Guido, M.; De Donno, A.; Bella, A.; Ciofi degli Atti, M.L.; Crovari, P.; Seroepidemiology Group. The epidemiology of Varicella Zoster infection in Italy. BMC Public Health 2008, 8, 372. Doi:10.1186/1471-2458-8-372.

3. Grose, C. Varicella vaccination of children in the United States: assessment after the first decade 1995-2005. J. Clin. Virol. 2005, 33, 89-95.

4. Heininger, U.; Seward, J.F. Varicella. Lancet 2006, 368, 1365-1376.

5. Burke, M.S. Herpes Zoster vaccine: clinical trial evidence and implications for medical practice. $J$. Am. Osteopath. Assoc. 2007, 107, S14-S18.

6. Thomas, S.L.; Hall A.J. What does epidemiology tell us about risk factors for herpes zoster? Lancet Infect. Dis. 2004, 4, 26-33.

7. Kempf, W.; Meylan, P.; Gerber, S.; Aebi, C.; Agosti, R.; Buchner, S.; Coradi, B.; Garweg, J.; Hirsch, H.; Kind, C.; Lauper, U.; Lautenschlager, S.; Reusser, P.; Ruef, C.; Wunderli, W.; Nadal, D. Swiss recommendations for the management of varicella zoster virus infections. Swiss Med. Wkly. 2007, 137, 239-251. 
8. Bowsher, D. The lifetime occurrence of Herpes Zoster and prevalence of post-herpetic neuralgia: a retrospective survey in an elderly population. Eur. J. Pain. 1999, 3, 335-342.

9. Bowsher, D. Factors influencing the features of postherpetic neuralgia and outcome when treated with tricyclics. Eur. J. Pain. 2003, 7, 1-7.

10. Jung, B.F.; Johnson, R.W.; Griffin, D.R.; Dworkin, R.H. Risk factors for postherpetic neuralgia in patients with herpes zoster. Neurology 2004, 62, 1545-1551.

11. Whitley, R.J.; Gnann, J.W., Jr. Herpes zoster in patients with human immunodeficiency virus infection - an ever-expanding spectrum of disease. Clin. Infect. Dis. 1995, 21, 989-990.

12. Marocco, A.; Assael, B.; Gabutti, G.; Guarino, A.; Lopalco, P.L.; Marchetti, F.; Ruggeri, F.M.; Titone, L.; Tozzi, A.E.; Vitali Rosati, G.; Zotti, C.; Franco, E. Hospitalisation associated with Rotavirus gastroenteritis in Italy, 2001-2003, evaluated by means of ICD9-CM diagnostic codes. Ig. Sanità Pubbl. 2006, 62, 215-224.

13. Ministro della Sanità. Decreto Ministeriale 28 Dicembre 1991. Istituzione della scheda di dimissione ospedaliera. Gazzetta Ufficiale 1992, 17, 13.

14. Gabutti, G.; Marsella, M.; Lazzara, C.; Fiumana, E.; Cavallaro, A.; Borgna-Pignatti, C. Epidemiology and burden of Rotavirus-associated hospitalizations in Ferrara, Italy. J. Prev. Med. Hyg. 2007, 48, 5-9.

15. Ministero della Sanità. Decreto Ministeriale 15 dicembre 1990. Sistema di sorveglianza sulle malattie infettive. Gazzetta Ufficiale 1991, 6, 8-18.

16. Di Luzio Paparatti, U.; Arpinelli, F.; Visonà, G. Herpes Zoster and its complications in Italy: an observational survey. J. Infect. 1999, 38, 116-120.

17. Eberti Gialloreti, L.; Divizia, M.; Pica, F.; Volpi, A. Analysis of the cost-effectiveness of varicella vaccine programmes based on an observational survey in the Latium region in Italy. Herpes 2005, $12,33-37$.

18. Di Legami, V.; Gianino, M.M.; Ciofi degli Atti, M.; Massari, M.; Migliardi, A.; Tomba, G.S.; Zotti, C.; Zoster Study Group. Epidemiology and costs of herpes zoster: background data to estimate the impact of vaccination. Vaccine 2007, 25, 7598-7604.

19. Volpi, A. Varicella immunization and Herpes Zoster. Herpes 2005, 12, 59.

20. Gross, G.; Schofer, H.; Wassilew, S.; Friese, K.; Timm, A.; Guthoff, R.; Pau, H.W.; Malin, J.P.; Wutzler, P.; Doerr, H.W. Herpes zoster guideline of the German Dermatology Society (DDG). J. Clin. Virol. 2003, 26, 277-289.

21. Dworkin R.H.; Schmader K.E. Treatment and prevention of postherpetic neuralgia. Clin. Pract. 2003, 36, 877-882.

22. Young, L. Post-herpetic neuralgia: a review of advances in treatment and prevention. J. Drugs Dermatol. 2006, 5, 938-941.

23. Levin, M.L.; Smith, J.G.; Kaufhold, R.M.; Barber, D.; Hayward, A.R.; Chan, C.Y.; Chan, I.S.; Li, D.J.; Wang, W.; Keller, P.M.; Shaw, A.; Silber, J.L.; Schlienger, K.; Chalikonda, I.; Vessey, S.J.; Caulfield, M.J. Decline in varicella-zoster virus (VZV)-specific cell-mediated immunity with increasing age and boosting with a high-dose VZV vaccine. JID 2003, 188, 1336-1344.

24. Oxman, M.N.; Levin, M.J.; Johnson, G.R.; Schmader, K.E.; Straus, S.E.; Gelb, L.D.; Arbeit, R.D.; Simberkoff, M.S.; Gershon, A.A.; Davis, L.E.; Weinberg, A.; Boardman, K.D.; Williams, H.M.; 
Zhang, J.H.; Peduzzi, P.N.; Beisel, C.E.; Morrison, V.A.; Guatelli, J.C.; Brooks, P.A.; Kauffman, C.A.; Pachucki, C.T.; Neuzil, K.M.; Betts, R.F.; Wright, P.F.; Griffin, M.R.; Brunell, P.; Soto, N.E.; Marques, A.R.; Keay, S.K.; Goodman, R.P.; Cotton, D.J.; Gnann, J.W.jr.; Loutit, J.; Holodniy, M.; Keitel, W.A.; Crawford, G.E.; Yeh, S.S.; Lobo, Z.; Toney, J.F.; Greenberg, R.N.; Keller, P.M.; Harbecke, R.; Hayward, A.R.; Irwin, M.R.; Kyriakides, T.C.; Chan, C.Y.; Chan, I.S.; Wang, W.W.; Annunziato, P.W.; Silber, J.L. Shingles Prevention Study Group. A vaccine to prevent herpes zoster and postherpetic neuralgia in older adults. NEJM 2005, 352, 2271-2284.

25. Oxman, M.N. Vaccination to prevent Herpes Zoster and postherpetic neuralgia. Hum. Vacc. 2007, 3, 64-68.

26. CDC. Prevention of Herpes Zoster. Recommendations of the Advisory Committee on immunization practices (ACIP). MMWR 2008, 57, 1-32.

(C) 2009 by the authors; licensee Molecular Diversity Preservation International, Basel, Switzerland. This article is an open-access article distributed under the terms and conditions of the Creative Commons Attribution license (http://creativecommons.org/licenses/by/3.0/). 